\title{
Effect of Mycorrhizal Inoculation at Different Salinity Levels on Root Coloniza- tion, Growth and Chlorophyll Content of Different Grape Rootstocks (Vitis spp)
}

\author{
${ }^{* 1}$ B.Y. Derbew , ${ }^{2}$ A.N. Mokashi,${ }^{2}$ C.P. Patil and ${ }^{2}$ R.V. Hegde \\ ${ }^{1}$ College of Agriculture, Jimma University, P.O.Box 307, Jimma, Ethiopia \\ Email: dbelew2002@yahoo.com \\ ${ }^{2}$ University of Agricultural Sciences, Dharwad 580 005, Karnataka, India
}

Accepted 25 $5^{\text {th }}$ August 2007

\begin{abstract}
A pot culture experiment was conducted during 2004-2006 at the College of Agriculture, University of Agricultural Sciences, Dharwad, to investigate effect of arbuscular mycorrhizal (AM) fungus (Glomus fasciculatum) inoculation at different salinity levels $(0.52,1.90,4.33,6.23$ and $7.94 \mathrm{dSm}^{-1}$ ) on root colonization, growth and chlorophyll content of four grape rootstocks (Salt Creek, Dogridge, St. George and 1613). The extent of AM response on root colonization, growth and chlorophyll content varied with rootstock species, and with the level of salinity. AM fungus inoculated plants showed significantly higher root colonization percentage, root volume, root length, number of leaves, leaf area, total dry weight, and chlorophyll content. Exposure to salinity stress resulted in decreased root colonization, chlorophyll content and growth of shoots on all rootstocks, but reduction in growth was greatest on St. George.
\end{abstract}

Keywords: grape, rootstock, salinity, mycorrhiza, root colonization, chlorophyll.

\section{INTRODUCTION}

Grape (Vitis spp.) is one of the most commercially grown important fruit crops in the world. In India grapes are cultivated at an extent of 40,000 hectares across the country with an estimated production of 1.2 million tonnes (Anonymous, 2003). Maharashtra, Andhra Pradesh, Karnataka and Tamil Nadu in western and southern India, and Punjab, Haryana and Uttar Pradesh in northern India, are the major grape growing states. Over 90 per cent of the area occupied by grape cultivation is found in semi arid regions of Maharashtra, northern Karnataka and Andhra Pradesh. In the last five to six years, grape productivity in these states has been constrained by water scarcity due to regular monsoon failure and soil salinity. Saltaffected soils cover an area of nearly $13.5 \mathrm{M}$ ha in India (Sharma et al., 2004) and 173 thousand ha in Karnataka (Sharma, 1998).

Salinity is an environmental stress that limits growth and development in plants. The response of plants to excess salt is complex and involves changes in their morphology, physiology and metabolism (Shannon et al., 1994). In arid and semi-arid regions of the world, limited rainfall, high evapotranspiration, high temperature and inadequate water management contribute to increase in soil salinity. In those areas, plant growth is severely affected by salinity through water deficit, salt-specific damages (Munns and Termaat, 1986) or oxidative stress (Hernandez et al., 1995). Plants' capacity to endure the effects of excessive salt in the root zone is the "salt tolerance" of plants. Plants vary in their response to soil salinity and the range of salt concentrations tolerated by crops varies greatly from species to species (Volkmar et al., 1998).

Arbuscular mycorrhizal (AM) fungi improve physiological processes, like water absorption capacity of plants by increasing root hydraulic conductivity and favourably adjusting the osmotic balance and composition of carbohydrates (Rosendahl and Rosendahl 1991). Thus, they mitigate the adverse effects of excess salt accumulation in the root (Dixon et al., 1993). An experiment was conducted to determine the effect of mycorrhizal inoculation at different salinity on root colonization, plant growth and chlorophyll content of grape rootstocks (Vitis spp).

*Corresponding author 


\section{MATERIALS AND METHODS}

Cuttings of four grape rootstocks viz., Dogridge, Salt Creek, St. George and 1613 were rooted in nursery beds at College of Agriculture, University of Agricultural Sciences, Dharwad (situated $15^{\circ}-26^{\prime}$ North latitude and $70^{\circ}$ 07' East longitude). Experimental design used was factorial CRD, with four replications, wherein a total of four plants per treatment were grown. The inoculation of AM fungus to grape cuttings was done in the nursery bed using five grams of inoculum ( $G$. fasciculatum) per cutting consisting of 19 infective propagules (chlamydospores) per gram of inoculum at five centimeters depth. After putting a thin layer of soil on the inoculum, grape cuttings of about 25-30 cm long, having four nodes, were placed and two buds of the cuttings covered with soil. Two months old rooted cuttings were transferred to a 6" 69 " size polythene bags and allowed to grow for four months (until they attained pencil size shoot girth). At this stage the rooted cuttings were removed from polythene bags and imposed to different levels of salinity $(0.52,1.90,4.33$, 6.23 and $7.94 \mathrm{dSm}^{-1}$ ), which were obtained from naturally salt affected soil in Gangawati
Agricultural Research Station of the University of Agricultural Sciences, Dharwad (Raichur district, Northern Karnataka). To maintain level of salinity, plants were given a measured quantity of water and kept under salinity stress condition for four months. Per cent root colonization was determined following the procedure outlined by Phillips and Hayman (1970). Leaf area was determined using Portable Area Meter, Model LI-3000A, LI-COR. Chlorophyll content of the leaves was determined following dimethyl sulfoxide (DMSO) method. At the end of the experimental period, plants were carefully removed from earthen pots and shoot and root parts were separated and the roots washed in water. In order to obtain dry weight of shoot and root, fresh tissues were dried at $70^{\circ} \mathrm{C}$ in an oven for 24 hours till constant weight was reached.

\section{RESULTS AND DISCUSSION}

Mycorrhizal inoculation and salinity stress had strong effects on growth, root colonization and leaf chlorophyll content. Growth parameters (number of leaves per vine, leaf area, root volume, root length and total dry weight) and per

Table 1. Effect of mycorrhizal inoculation and different salinity levels on root colonization, root volume and root length of grape rootstocks

\begin{tabular}{|c|c|c|c|c|c|c|c|c|c|c|c|c|c|c|c|c|c|c|c|}
\hline \multirow{3}{*}{\multicolumn{2}{|c|}{$\begin{array}{l}\text { AMF Root } \\
\text { inocu stocks } \\
\text { lation }\end{array}$}} & \multicolumn{6}{|c|}{ Root colonization $(\%)$} & \multicolumn{6}{|c|}{ Root volume (cc) } & \multicolumn{6}{|c|}{ Root length (cm) } \\
\hline & & \multicolumn{6}{|c|}{ Salinity levels } & \multicolumn{6}{|c|}{ Salinity levels } & \multicolumn{6}{|c|}{ Salinity levels } \\
\hline & & So & S1 & $\mathbf{S 2}$ & S3 & S4 & Mean & S0 & S1 & $\mathbf{S 2}$ & S3 & S4 & Mean & So & S1 & $\mathbf{S 2}$ & S3 & S4 & Mean \\
\hline \multirow[t]{5}{*}{$\overline{\mathrm{M} 0}$} & $\mathrm{R} 1$ & 45.00 & 40.00 & 40.00 & 36.00 & 31.00 & 38.40 & 24.00 & 21.22 & 20.36 & 18.00 & 15.25 & 19.76 & 90.30 & 79.30 & 47.27 & 46.28 & 40.28 & 60.69 \\
\hline & $\mathrm{R} 2$ & 48.00 & 43.00 & 42.00 & 40.00 & 35.00 & 34.60 & 21.17 & 15.18 & 15.12 & 14.50 & 12.28 & 15.65 & 45.42 & 38.30 & 34.32 & 23.27 & 20.25 & 32.31 \\
\hline & R3 & 44.00 & 43.00 & 43.00 & 41.00 & 36.00 & 34.20 & 30.33 & 24.17 & 23.19 & 21.17 & 18.25 & 23.42 & 60.30 & 40.30 & 34.30 & 22.30 & 19.67 & 35.37 \\
\hline & R4 & 44.00 & 42.00 & 42.00 & 41.00 & 36.00 & 33.80 & 26.95 & 18.25 & 18.22 & 15.08 & 14.47 & 18.59 & 50.28 & 41.28 & 32.33 & 30.27 & 26.34 & 36.10 \\
\hline & Mean & 45.25 & 42.00 & 41.75 & 39.50 & 34.50 & 35.25 & 25.61 & 19.70 & 19.22 & 17.19 & 15.06 & 19.36 & 61.58 & 49.80 & 37.05 & 30.53 & 26.64 & 41.12 \\
\hline \multirow[t]{12}{*}{ M1 } & R1 & 52.00 & 51.00 & 49.00 & 45.00 & 40.00 & 39.40 & 30.15 & 24.17 & 23.19 & 18.15 & 17.00 & 22.53 & 81.30 & 74.28 & 67.28 & 59.28 & 51.60 & 66.75 \\
\hline & $\mathrm{R} 2$ & 66.00 & 63.00 & 62.00 & 57.00 & 52.00 & 49.60 & 24.22 & 18.05 & 17.32 & 15.17 & 12.22 & 17.39 & 46.27 & 32.28 & 28.30 & 20.30 & 17.67 & 28.96 \\
\hline & R3 & 65.00 & 62.00 & 61.00 & 59.00 & 54.00 & 49.40 & 35.12 & 32.82 & 30.18 & 21.02 & 20.16 & 27.86 & 65.13 & 45.23 & 43.28 & 27.30 & 23.15 & 40.82 \\
\hline & $\mathrm{R} 4$ & 78.00 & 78.00 & 76.00 & 72.00 & 67.00 & 60.80 & 33.00 & 30.12 & 24.17 & 18.22 & 17.48 & 24.60 & 62.33 & 48.27 & 36.25 & 25.28 & 22.01 & 38.83 \\
\hline & Mean & 65.25 & 63.50 & 62.00 & 58.25 & 53.25 & 49.80 & 30.62 & 26.29 & 23.71 & 18.14 & 16.71 & 23.09 & 63.76 & 50.02 & 43.78 & 33.04 & 28.61 & 43.84 \\
\hline & \multicolumn{19}{|c|}{ For comparison of rootstocks and salinity } \\
\hline & $\mathrm{R} 1$ & 48.50 & 45.50 & 44.50 & 40.50 & 35.50 & 38.90 & 27.08 & 22.69 & 21.77 & 18.08 & 16.13 & 21.15 & 85.80 & 76.79 & 57.28 & 52.78 & 45.94 & 63.72 \\
\hline & $\mathrm{R} 2$ & 57.00 & 53.00 & 52.00 & 48.50 & 43.50 & 42.10 & 22.69 & 16.62 & 16.22 & 14.84 & 12.25 & 16.52 & 45.84 & 35.29 & 31.31 & 21.78 & 18.96 & 30.64 \\
\hline & $\mathrm{R} 3$ & 54.50 & 52.50 & 52.00 & 50.00 & 45.00 & 41.80 & 32.73 & 28.49 & 26.69 & 21.09 & 19.21 & 25.64 & 62.72 & 42.77 & 38.79 & 24.80 & 21.41 & 38.10 \\
\hline & $\mathrm{R} 4$ & 61.00 & 60.00 & 59.00 & 56.50 & 51.50 & 47.30 & 29.98 & 24.18 & 21.19 & 16.65 & 15.98 & 21.60 & 56.31 & 44.78 & 34.29 & 27.78 & 24.17 & 37.46 \\
\hline & \multirow[t]{2}{*}{ Mean } & 55.25 & 52.75 & 51.88 & 48.88 & 43.88 & 42.53 & 28.12 & 23.00 & 21.47 & 17.66 & 15.89 & 21.23 & 62.67 & 49.91 & 40.42 & 31.79 & 27.62 & 42.48 \\
\hline & & S.Em \pm & CD $5 \%$ & & & & & & & S.Em \pm & CD $5 \%$ & & & & & \multicolumn{3}{|c|}{ S.Em \pm CD $5 \%$} & \\
\hline & $\sqrt{11}$ & 0.61 & 1.72 & & & & & \multicolumn{2}{|c|}{ M } & 0.30 & 0.83 & & & & M & 0.42 & 1.20 & & \\
\hline & $\mathrm{R}$ & 0.86 & 2.43 & & & & & \multicolumn{2}{|c|}{$\mathrm{R}$} & 0.42 & 1.18 & & & & $\mathrm{R}$ & 0.60 & 1.69 & & \\
\hline & $S$ & 0.96 & 2.72 & & & & & \multicolumn{2}{|c|}{ S } & 0.47 & 1.32 & & & & $S$ & 0.67 & 1.89 & & \\
\hline & x R & 1.22 & 3.44 & & & & & \multicolumn{2}{|c|}{$M \times R$} & 0.59 & 1.67 & & & $M x$ & x R & 0.85 & 2.39 & & \\
\hline & x S & 1.36 & 3.84 & & & & & \multicolumn{2}{|c|}{$M \times S$} & 0.66 & 1.86 & & & $\mathrm{Mx}_{\mathrm{x}}$ & x S & 0.95 & 2.67 & & \\
\hline & x S & 1.93 & 5.43 & & & & & \multicolumn{2}{|c|}{$\mathrm{R} \times \mathrm{S}$} & 0.94 & NS & & & $\mathrm{R} x$ & $x \mathrm{~S}$ & 1.34 & 3.78 & & \\
\hline $\mathrm{Mx}$ & $\mathrm{R} \times \mathrm{S}$ & 2.73 & 7.68 & & & & & \multicolumn{2}{|c|}{$M \times R \times S$} & 1.32 & NS & & & $\mathrm{M} \times \mathrm{R}$ & $\mathrm{R} \times \mathrm{S}$ & 1.90 & 5.35 & & \\
\hline
\end{tabular}

Mycorrhizal treatment (M): M0=Uninoculated, M1=Inoculated,

Rootstocks (R): R1 = Dogridge, R2=St. George, R3=Salt Creek, R4=1613; Salinity levels (S): S0= Control $(0.52), \mathrm{S} 1=1.90$, $\mathrm{S} 2=4.33, \mathrm{~S} 3=6.23, \mathrm{~S} 4=7.94 \mathrm{dSm}^{-1}$ 
Table 2. Effect of mycorrhizal inoculation and different salinity levels on number of leaves, leaf area and total dry weight of grape rootstocks

\begin{tabular}{|c|c|c|c|c|c|c|c|c|c|c|c|c|c|c|c|c|c|c|c|}
\hline \multirow{3}{*}{$\begin{array}{r}\text { AMF } \\
\text { inocu } \\
\text { lation. }\end{array}$} & \multirow{3}{*}{$\begin{array}{c}\text { Root } \\
\text { stocks }\end{array}$} & \multicolumn{6}{|c|}{ Number of leaves } & \multicolumn{6}{|c|}{ Leaf area $\left(\mathrm{cm}^{2}\right)$} & \multicolumn{6}{|c|}{ Total dry weight (g) } \\
\hline & & \multicolumn{6}{|c|}{ Salinity levels } & \multicolumn{6}{|c|}{ Salinity levels } & \multicolumn{6}{|c|}{ Salinity levels } \\
\hline & & S0 & S1 & $\mathbf{S 2}$ & S3 & $\mathbf{S 4}$ & Mean & S0 & S1 & S2 & S3 & S4 & Mean & S0 & S1 & S2 & $\mathbf{S 3}$ & S4 & Mean \\
\hline \multirow[t]{5}{*}{$\overline{\mathrm{M} 0}$} & $\mathrm{R} 1$ & 15.33 & 12.33 & 12.00 & 8.67 & 5.67 & 10.80 & 19.20 & 16.39 & 14.13 & 13.61 & 12.55 & 15.18 & 14.16 & 9.02 & 8.01 & 6.22 & 5.68 & 8.62 \\
\hline & $\mathrm{R} 2$ & 13.67 & 12.67 & 11.67 & 11.33 & 7.33 & 11.33 & 18.59 & 13.50 & 11.01 & 10.56 & 9.34 & 12.60 & 7.41 & 3.94 & 3.61 & 2.80 & 2.36 & 4.02 \\
\hline & R3 & 16.00 & 14.33 & 13.00 & 11.00 & 7.33 & 12.33 & 27.91 & 26.55 & 22.00 & 15.51 & 14.55 & 21.30 & 18.31 & 14.17 & 11.99 & 9.39 & 8.73 & 12.52 \\
\hline & $\mathrm{R} 4$ & 19.00 & 17.00 & 16.67 & 9.00 & 5.67 & 13.47 & 35.25 & 25.78 & 23.90 & 20.43 & 19.72 & 25.02 & 11.92 & 8.84 & 7.04 & 5.23 & 4.74 & 7.55 \\
\hline & Mean & 16.00 & 14.08 & 13.33 & 10.00 & 6.50 & 11.98 & 25.24 & 20.56 & 17.76 & 15.03 & 14.04 & 18.52 & 12.95 & 8.99 & 7.66 & 5.91 & 5.38 & 8.18 \\
\hline \multirow[t]{11}{*}{ M1 } & R1 & 20.67 & 20.33 & 18.00 & 14.67 & 9.67 & 16.67 & 29.42 & 22.77 & 20.88 & 20.42 & 19.71 & 22.64 & 14.66 & 12.32 & 9.73 & 7.06 & 6.50 & 10.05 \\
\hline & $\mathrm{R} 2$ & 16.00 & 14.33 & 12.33 & 10.33 & 6.67 & 11.93 & 28.60 & 20.29 & 19.60 & 11.08 & 9.88 & 17.89 & 10.88 & 6.80 & 4.51 & 4.10 & 3.62 & 5.98 \\
\hline & R3 & 16.67 & 15.67 & 15.67 & 9.33 & 6.33 & 12.73 & 53.41 & 43.35 & 26.79 & 22.70 & 22.11 & 33.67 & 20.04 & 16.75 & 12.70 & 11.62 & 10.91 & 14.40 \\
\hline & $\mathrm{R} 4$ & 23.00 & 18.00 & 17.33 & 16.33 & 10.67 & 17.07 & 37.91 & 34.84 & 30.64 & 27.67 & 27.35 & 31.68 & 14.78 & 11.98 & 8.53 & 5.60 & 5.09 & 9.20 \\
\hline & Mean & 19.08 & 17.08 & 15.83 & 12.67 & 8.33 & 14.60 & 37.34 & 30.31 & 24.48 & 20.47 & 19.76 & 26.47 & 15.09 & 11.96 & 8.87 & 7.10 & 6.53 & 9.91 \\
\hline & \multicolumn{19}{|c|}{ For comparison of rootstocks and salinity } \\
\hline & $\mathrm{R} 1$ & 18.00 & 16.33 & 15.00 & 11.67 & 7.67 & 13.73 & 24.31 & 19.58 & 17.51 & 17.01 & 16.13 & 18.91 & 14.41 & 10.67 & 8.87 & 6.64 & 6.09 & 9.34 \\
\hline & $\mathrm{R} 2$ & 14.83 & 13.50 & 12.00 & 10.83 & 7.00 & 11.63 & 23.60 & 16.90 & 15.31 & 10.82 & 9.61 & 15.25 & 9.15 & 5.37 & 4.06 & 3.45 & 2.99 & 5.00 \\
\hline & R3 & 16.33 & 15.00 & 14.33 & 10.17 & 6.83 & 12.53 & 40.66 & 34.95 & 24.40 & 19.10 & 18.33 & 27.49 & 19.18 & 15.46 & 12.35 & 10.51 & 9.82 & 13.46 \\
\hline & $\mathrm{R} 4$ & 21.00 & 17.50 & 17.00 & 12.67 & 8.17 & 15.27 & 36.58 & 30.31 & 27.27 & 24.05 & 23.54 & 28.35 & 13.35 & 10.41 & 7.79 & 5.42 & 4.92 & 8.38 \\
\hline & Mean & 17.54 & 15.58 & 14.58 & 11.33 & 7.42 & 13.29 & 31.29 & 25.43 & 21.12 & 17.75 & 16.90 & 22.50 & 14.02 & 10.48 & 8.27 & 6.50 & 5.95 & 9.04 \\
\hline \multicolumn{8}{|c|}{ S.Em \pm CD $5 \%$} & \multicolumn{6}{|c|}{ S.Em \pm CD $5 \%$} & \multicolumn{6}{|c|}{ S.Em \pm CD 5\% } \\
\hline & И & 0.43 & 1.20 & & & & & \multicolumn{2}{|c|}{ M } & 0.25 & 0.72 & & & \multicolumn{2}{|c|}{ M } & 0.02 & 0.05 & & \\
\hline & $\mathrm{R}$ & 0.60 & 1.70 & & & & & \multicolumn{2}{|c|}{$\mathrm{R}$} & 0.36 & 1.01 & & & \multicolumn{2}{|c|}{$\mathrm{R}$} & 0.02 & 0.07 & & \\
\hline & $S$ & 0.67 & 1.90 & & & & & \multicolumn{2}{|c|}{ S } & 0.40 & 1.13 & & & \multicolumn{2}{|c|}{$\mathrm{S}$} & 0.03 & 0.08 & & \\
\hline & $x \mathrm{R}$ & 0.85 & 2.40 & & & & & \multicolumn{2}{|c|}{ M x R } & 0.51 & 1.43 & & & \multicolumn{2}{|c|}{$M \times R$} & 0.03 & 0.10 & & \\
\hline & x S & 0.95 & 2.68 & & & & & \multicolumn{2}{|c|}{$M \times S$} & 0.57 & 1.60 & & & \multicolumn{2}{|c|}{$M \times S$} & 0.04 & 0.11 & & \\
\hline & x S & 1.35 & 3.79 & & & & & \multicolumn{2}{|c|}{$R \times S$} & 0.80 & 2.26 & & & \multicolumn{2}{|c|}{$R \times S$} & 0.05 & 0.15 & & \\
\hline $\mathrm{Mx}$ & $\mathrm{R} \times \mathrm{S}$ & 1.91 & $\mathrm{NS}$ & & & & & $\mathrm{Mx}$ & $\mathrm{R} \times \mathrm{S}$ & 1.14 & 3.20 & & & $\mathrm{M} \times \mathrm{F}$ & $\mathrm{R} \times \mathrm{S}$ & 0.08 & 0.21 & & \\
\hline
\end{tabular}

Mycorrhizal treatment (M): M0=Uninoculated, M1=Inoculated,

Rootstocks (R): R1 = Dogridge, R2=St. George, R3=Salt Creek, R4=1613; Salinity levels (S): S0=Control (0.52), S1=1.90,

$\mathrm{S} 2=4.33, \mathrm{~S} 3=6.23, \mathrm{~S} 4=7.94 \mathrm{dSm}^{-1}$

Table 3. Effect of mycorrhizal inoculation and different salinity levels on chlorophyll 'a', 'b', and total chlorophyll content of grape rootstocks

\begin{tabular}{|c|c|c|c|c|c|c|c|c|c|c|c|c|c|c|c|c|c|c|c|}
\hline \multirow{3}{*}{$\begin{array}{l}\text { AMF } \\
\text { inocu } \\
\text { lation }\end{array}$} & \multirow[t]{3}{*}{$\begin{array}{l}\text { Root- } \\
\text { stocks }\end{array}$} & \multicolumn{6}{|c|}{ Chlorophyll ' a' content (mg g fr. wt. $\left.{ }^{-1}\right)$} & \multicolumn{6}{|c|}{ Chlorophyll 'b' content (mg g fr. wt.' } & \multicolumn{6}{|c|}{ Total chlorophyll content (mg g fr. wt. $_{11}^{-}$} \\
\hline & & \multicolumn{6}{|c|}{ Salinity levels } & \multicolumn{6}{|c|}{ Salinity levels } & \multicolumn{6}{|c|}{ Salinity levels } \\
\hline & & S0 & S1 & S2 & $\mathbf{S 3}$ & $\mathbf{S 4}$ & Mean & S0 & S1 & $\mathbf{S 2}$ & $\mathbf{S 3}$ & S4 & Mean & S0 & S1 & S2 & $\mathbf{S 3}$ & S4 & Mean \\
\hline \multirow[t]{5}{*}{ M0 } & R1 & 1.52 & 1.47 & 1.35 & 0.98 & 0.58 & 1.18 & 0.78 & 0.75 & 0.61 & 0.40 & 0.35 & 0.63 & 2.30 & 2.22 & 1.95 & 1.38 & 0.93 & 1.76 \\
\hline & $\mathrm{R} 2$ & 1.31 & 1.23 & 1.20 & 0.77 & 0.74 & 1.05 & 0.56 & 0.52 & 0.47 & 0.42 & 0.34 & 0.47 & 1.87 & 1.75 & 1.21 & 1.62 & 1.11 & 1.51 \\
\hline & R3 & 1.63 & 1.53 & 1.38 & 1.21 & 0.72 & 1.29 & 1.08 & 0.86 & 0.65 & 0.55 & 0.49 & 0.79 & 2.71 & 2.39 & 2.04 & 1.75 & 1.22 & 2.02 \\
\hline & $\mathrm{R} 4$ & 1.64 & 1.56 & 1.53 & 1.22 & 0.73 & 1.34 & 1.14 & 1.01 & 0.86 & 0.56 & 0.51 & 0.89 & 2.78 & 2.57 & 2.39 & 1.79 & 1.24 & 2.15 \\
\hline & Mean & 1.52 & 1.45 & 1.36 & 1.05 & 0.69 & 1.22 & 0.89 & 0.79 & 0.65 & 0.48 & 0.42 & 0.70 & 2.42 & 2.23 & 1.90 & 1.63 & 1.12 & 1.86 \\
\hline \multirow[t]{19}{*}{ M1 } & R1 & 1.55 & 1.50 & 1.38 & 1.32 & 0.79 & 1.31 & 0.83 & 0.76 & 0.64 & 0.60 & 0.55 & 0.71 & 2.37 & 2.26 & 2.01 & 1.91 & 1.34 & 1.98 \\
\hline & $\mathrm{R} 2$ & 1.53 & 1.50 & 1.33 & 1.32 & 0.80 & 1.29 & 0.80 & 0.77 & 0.60 & 0.59 & 0.54 & 0.69 & 2.33 & 2.27 & 1.92 & 1.91 & 1.34 & 1.95 \\
\hline & R3 & 1.65 & 1.56 & 1.51 & 1.44 & 0.87 & 1.41 & 1.33 & 0.89 & 0.83 & 0.69 & 0.64 & 0.94 & 2.98 & 2.45 & 2.34 & 2.14 & 1.51 & 2.28 \\
\hline & $\mathrm{R} 4$ & 1.64 & 1.58 & 1.54 & 1.55 & 0.88 & 1.44 & 1.17 & 0.99 & 0.91 & 0.89 & 0.86 & 0.99 & 2.80 & 2.57 & 2.45 & 2.44 & 1.74 & 2.40 \\
\hline & Mean & 1.59 & 1.53 & 1.44 & 1.41 & $(0.83)$ & 1.36 & 1.03 & 0.85 & 0.74 & 0.69 & 0.65 & 0.83 & 2.62 & 2.39 & 2.18 & 2.10 & 1.48 & 2.15 \\
\hline & \multicolumn{19}{|c|}{ For comparison of rootstocks and salinity } \\
\hline & DG & 1.53 & 1.48 & 1.36 & 1.15 & 0.69 & 1.24 & 0.80 & 0.76 & 0.62 & 0.50 & 0.45 & 0.67 & 2.33 & 2.24 & 1.98 & 1.65 & 1.13 & 1.87 \\
\hline & SG & 1.42 & 1.37 & 1.26 & 1.05 & 0.77 & 1.17 & 0.68 & 0.65 & 0.53 & 0.50 & 0.44 & 0.58 & 2.10 & 2.01 & 1.57 & 1.77 & 1.23 & 1.73 \\
\hline & $\mathrm{SC}$ & 1.64 & 1.54 & 1.45 & 1.33 & 0.79 & 1.35 & 1.21 & 0.88 & 0.74 & 0.62 & 0.57 & 0.86 & 2.85 & 2.42 & 2.19 & 1.95 & 1.36 & 2.15 \\
\hline & 1613 & 1.64 & 1.57 & 1.53 & 1.39 & 0.81 & 1.39 & 1.16 & 1.00 & 0.88 & 0.73 & 0.68 & 0.94 & 2.79 & 2.57 & 2.42 & 2.11 & 1.49 & 2.28 \\
\hline & Mean & 1.56 & 1.49 & 1.40 & 1.23 & 0.76 & 1.29 & 0.96 & 0.82 & 0.70 & 0.59 & 0.54 & 0.76 & 2.52 & 2.31 & 2.04 & 1.87 & 1.30 & 2.01 \\
\hline & & S.Em \pm & CD $5 \%$ & & & & & & & S.Em \pm & CD $5 \%$ & & & & & S.Em \pm & CD 5\% & & \\
\hline & M & 0.03 & 0.07 & & & & & \multicolumn{2}{|c|}{ M } & 0.004 & 0.011 & & & \multicolumn{2}{|c|}{ M } & 0.03 & 0.08 & & \\
\hline & $\mathrm{R}$ & 0.04 & 0.10 & & & & & \multicolumn{2}{|c|}{$\mathrm{R}$} & 0.005 & 0.015 & & & \multicolumn{2}{|c|}{$\mathrm{R}$} & 0.04 & 0.11 & & \\
\hline & $\mathrm{S}$ & 0.04 & 0.12 & & & & & \multicolumn{2}{|c|}{$\mathrm{S}$} & 0.006 & 0.017 & & & \multicolumn{2}{|c|}{$\mathrm{S}$} & 0.05 & 0.13 & & \\
\hline & $M \times R$ & 0.05 & 0.15 & & & & & \multirow{2}{*}{\multicolumn{2}{|c|}{$\begin{array}{l}M \times R \\
M \times S\end{array}$}} & 0.008 & 0.022 & & & \multirow{2}{*}{\multicolumn{2}{|c|}{$\begin{array}{l}M \times R \\
M \times S\end{array}$}} & 0.06 & NS & & \\
\hline & $M \times S$ & 0.06 & NS & & & & & M & & 0.009 & 0.024 & & & & & 0.06 & NS & & \\
\hline & $R \times S$ & 0.08 & 0.23 & & & & & \multicolumn{2}{|c|}{$\mathrm{R} \times \mathrm{S}$} & 0.012 & 0.034 & & & \multirow{2}{*}{\multicolumn{2}{|c|}{$\begin{array}{c}R \times S \\
M \times R \times S\end{array}$}} & 0.09 & 0.25 & & \\
\hline & $M \times R \times S$ & 0.12 & 0.33 & & & & & & & 0.017 & 0.049 & & & & & 0.13 & NS & & \\
\hline
\end{tabular}

Mycorrhizal treatment (M): M0=Uninoculated, M1=Inoculated,

Rootstocks (R): R1 = Dogridge, R2=St. George, R3=Salt Creek, R4=1613; Salinity levels (S): S0= Control (0.52), S1= 1.90, S2= $4.33, \mathrm{~S} 3=6.23, \mathrm{~S} 4=7.94 \mathrm{dSm}^{-1}$ 
cent root colonization decreased in all the rootstocks with increase in salinity stress from 0.52 to $7.94 \mathrm{dSm}^{-1}$. Mycorrhiza inoculated plants recorded significantly higher root colonization percentage, root volume and root length (Table 1), number of leaves per vine, leaf area and total dry weight (Table 2), and chlorophyll content (Table 3) compared to non-mycorrhizal plants.

Several greenhouse studies showed that grapevines inoculated with indigenous AM fungi had higher pruning weights and root weights (Linderman and Davis, 2001), and more compact, highly branched roots than nonmycorrhizal grapevines (Schellenbaum et al., 1991). Munns and Termaat (1986) suggested that growth inhibition in the long term exposure to increased salinity condition was related to lower photosynthetic area which will eventually become too low to support continuing growth. Munns (1993) proposed that accumulation of salt in the old leaves accelerated their death, and loss of these leaves decreased the supply of carbohydrates or growth hormones to meristematic regions, thereby inhibiting growth. Zekri (1991) concluded that salinity reduced shoot growth by suppressing leaf initiation and expansion as well as internode growth and by accelerating leaf abscission. The present study showed that salinity treatment caused significant decreases in leaf number of shoots. Decreases in the number of leaves were not only related to the growth inhibiting effects of salt, but also to the injurious effects of salt due to defoliation of the damaged leaves. Ramanujalu et al. (1993) observed gradual decrease in the contents of chlorophyll ' $a$ ' and chlorophyll ' $b$ ' with increase in the salt intensity in mulberry, wherein relatively higher rate of depletion was found with chlorophyll ' $a$ ' than chlorophyll ' $b$ '.

\section{REFERENCES}

Anonymous (2003) FAO bul. st. 4(2): 114-115.

Dixon, R.K., Garg, V.K. and Rao, M.V. (1993) Inoculation of Leucaena and Prosopis seedlings with Glomus and Rhizobium species in saline soil: rhizosphere relations and seedlings growth. Arid Soil Research Rehabilitation, 7:133-144.

Hernandez, J.A., Olmos, E., Corpas, F.J., Sevilla, F. and del Rio, L.A. (1995) Saltinduced oxidative stress in chloroplasts of pea plants. $P l$. Sci. 105:151-167.
Linderman, R.G. and Davis, A.A. (2001) Comparative response of selected grapevine rootstocks and cultivars to inoculation with different mycorrhizal fungi. Amer. J. Enol. Viticul. 52:8-11.

Munns, R. (1993) Physiological processes limiting plant growth in saline soils: some dogmas and hypotheses. Pl. Cell Environ. 16:15-24.

Munns, R. and Termaat, A. (1986) Wholeplant responses to salinity. Aust. J. Pl. Physiol. 13:143-160.

Philips, J.M. and Hayman, D.S. (1970) Improved procedures for clearing roots and staining parasitic and vesicular-arbuscular mycorrihizal fungi for rapid assessment of infection. Transac. Brit. Mycol. Soci. 55:158-161.

Ramanujalu, S., Veeranjaneyulu, K. and Sudhakar, C. (1993) Physiological changes induced by $\mathrm{NaCl}$ in mulberry var. Mysore local. Ind. J. Pl. Phys. 36:273-275.

Rosendahl, C.N. and Rosendahl, S. (1991) Infl uence of vesicular arbuscular mycorrhizal fungi (Glomus sp.) on the response of cucu mber (Cucumis sativus) to salt stress. Envir onment of Experimental Botany, 31: 313318.

Schellenbaum, L., Berta, G., Ravolanirina, F., Tisserant, B., Gianinazzi, S. and Fitter, A. H. (1991) Influence of endomycorrhizal inf ection on root morphology in a microprop agated woody plant species (Vitis vinifera L.). Ann. Bot. 68:135-141.

Shannon, M.C., Grieve, C.M. and Francois, L.E. (1994) Whole-plant response to salinity. In "Plant-Environment Interactions" (R.E. Wilkinson, Ed.), Dekker, New York, pp. 199-244.

Sharma, R.C. (1998) Nature, extent and classification, In: Agricultural Salinity Management in India. Central Soil Salinity Research Institute, Kernal.

Sharma, S.S., Totawat, K.L. and Shyampura, R.L. (2004) Characterization and Classification of Salt-affected Soils of Southern Rajasthan. J. Ind. Soc. Soil Sci. 52(3): 209214.

Volkmar, K.M., Hu Y. and Steppuhn, H. (1998) Physiological responses of plants to salinity: a review. Can. J. Pl. Sci. 78: 19-27.

Zekri, M. (1991) Effects of $\mathrm{NaCl}$ on growth and physiology of sour orange and Cleopatra mandarin seedlings. Sci. Hortic. 47: 305-315. 